\title{
L'ENTREPRISE RESPONSABLE DANS LA TRANSITION ÉCOLOGIQUE
}

\author{
Corinne Gendron, René Audet
}

ESKA | «Revue de l’organisation responsable »

2011/2 Vol. 6 | pages 60 à 60

ISSN 1951-0187

ISBN 9782747220118

Article disponible en ligne à l'adresse :

https://www.cairn.info/revue-de-l-organisation-responsable-2011-2-page-60.htm

Distribution électronique Cairn.info pour ESKA.

(C) ESKA. Tous droits réservés pour tous pays.

La reproduction ou représentation de cet article, notamment par photocopie, n'est autorisée que dans les limites des conditions générales d'utilisation du site ou, le cas échéant, des conditions générales de la licence souscrite par votre établissement. Toute autre reproduction ou représentation, en tout ou partie, sous quelque forme et de quelque manière que ce soit, est interdite sauf accord préalable et écrit de l'éditeur, en dehors des cas prévus par la législation en vigueur en France. Il est précisé que son stockage dans une base de données est également interdit. 


\section{L'ENTREPRISE RESPONSABLE DANS LA TRANSITION ÉCOLOGIQUE}

La Revue de l'Organisation Responsable (ROR) lance un appel à contributions pour son volume 8 $\mathrm{n}^{\circ} 2$ qui portera sur L'entreprise responsable dans la transition écologique.

Date limite de soumission : 31 mars 2013

Sous la direction de Corinne GENDRON et René AUDET,

professeurs, Chaire de responsabilité sociale et de développement durable

Le thème de la " la transition vers l'économie verte " fait aujourd'hui irruption dans l'espace public au côté de la " transition vers une économie sobre en carbone ", de la transition vers la durabilité » ou encore de la " transition juste ». Notamment porté par des organisations internationales telles que le Programme des Nations unies pour l'environnement (PNUE), l'Organisation pour la coopération et le développement économique (OCDE) et l'Agence internationale de l'énergie (AIE), ce nouveau discours est appelé à faire l'objet d'une mise en œuvre croissante dans les politiques nationales et infranationales. Il stipule notamment que les gouvernements devraient mettre en place un interventionnisme "vert " fondé sur des mesures incitatives qui auraient pour effet de modifier le calcul du risque lié à l'investissement dans les écoinnovations. Cet interventionnisme favoriserait les entreprises engagées envers le développement durable et entrainerait un mouvement plus large de modernisation économique aboutissant sur une transition vers une nouvelle économie verte.

Parallèlement à ce discours, de nombreux acteurs proposent des initiatives de villes, villages ou quartiers « en transition " et formulent une vision plus sociale et écologiste de la transition. Ici, c'est la communauté, les organisations locales et les citoyens qui sont considérés comme les principaux moteurs de la transition. L'entreprise y trouve un rôle dans la mesure où elle est à "l'échelle humaine ", comme c'est le cas des entreprises d'économie sociale et solidaires.

Enfin, le jeune courant interdisciplinaire des sustainability transitions studies se donne l'objectif d'analyser les transformations profondes à long terme des structures socioéconomiques et technologiques des sociétés contemporaines et se propose d'élaborer des méthodes pour " gérer » ou " piloter » les transitions sociotechniques. L'entreprise apparaît ici parfois comme un acteur capable de déverrouiller les " régimes sociotechniques " qui perpétuent la crise écologique, et parfois comme institution intrinsèquement constituante de ces régimes La transition apparait donc aujourd'hui à la fois comme un discours sur la réforme économique "verte ", comme un ensemble de pratiques et de méthodes visant la conduite du changement, et comme une approche théorique dédiée à la compréhension des transformations macrosociologiques qui pourraient mener à un monde plus écologique. Dans chaque cas, l'entreprise s'y trouve représentée dans des rôles divers et qui nécessitent des analyses approfondies. Dans ce contexte, les auteurs qui désirent contribuer au numéro sur L'entreprise responsable dans la transition écologique sont invités à aborder les thèmes suivants (non exclusifs) :

La mise en place d'un interventionnisme vert, fondé sur des incitatifs à l'investissement dans les éco-innovations, peut-elle véritablement entraîner une transition ? Parallèlement, cet interventionnisme implique-t-il une nouvelle responsabilité sociale des entreprises ?

Comment l'économie sociale et solidaire peut-elle s'inscrire dans un processus de transition au niveau local ? Comment peut-elle s'ancrer dans des processus de pilotage collectif de l'économie et de l'innovation verte?

Quelles formes d'entreprise et d'organisation sont le plus à même de réussir à transformer les structures socioéconomiques, les normes dominantes et les réseaux technologiques qui constituent les régimes sociotechniques ? L'entreprise responsable est-elle davantage un élément de verrouillage des régimes sociotechniques, ou bien un facteur de transformation de ces régimes?

Quelles approches théoriques permettraient de mieux comprendre et appréhender les transitions et les divers niveaux qu'elles comportent (économie globale, communautés, régimes sociotechniques, etc.) ?

La Revue acceptera des articles écrits dans l'une ou l'autre des deux langues. Chaque article écrit en français fera l'objet d'un résumé en anglais de façon que les mots clés et les résultats apparaissent clairement au lecteur anglophone et réciproquement chaque article écrit en anglais fera l'objet de la même présentation en français.

Les auteurs sont priés de respecter les recommandations cijointes concernant les règles de présentation des manuscrits

Les auteurs doivent suggérer deux évaluateurs indépendants susceptibles d'évaluer les articles de ce numéro. 